\title{
Design of part family robust-to-production plan variations based on quantitative manufacturability evaluation
}

\author{
Byungwoo Lee, Kazuhiro Saitou
}

\begin{abstract}
This paper presents a systematic method for designing part families whose production costs are insensitive to changes in production plans due to market demand fluctuations. A unified feature-based representation of functional geometry and manufacturability has been developed to manipulate and evaluate part designs. Based on this information and production plans for multiple periods, an optimization-based method provides alternative part designs. The manufacturability of the part designs is quantitatively estimated by the facility cost of the manufacturing system best configured for a given part family and the average cycle time estimated by the discrete event simulation of production scenarios. Redesign suggestions are made on datum definitions of the original parts. Two case studies of a family of prismatic parts and that of turned parts are given to demonstrate the effectiveness of the proposed method.
\end{abstract}

Keywords Part family, Design optimization, Design for manufacturing, Genetic algorithm

\section{1}

\section{Introduction}

Design for manufacturing (DFM) methods have been widely applied to various decisions throughout the design process such as the choice of material, shape, tolerances, standardization and assembly design (Bralla 1999; Boothroyd et al. 1999). According to van Vliet et al. (1999), there are three phases in manufacturability evaluation: verification, quantification and optimization. Among those three phases, methods for the second and third phase, especially for multiple products, are not well established compared with their counterparts for a single product. This is partly due to the difficulty in evaluating the

Received: 9 July 2001 / Revised: 18 June 2002

Accepted: 21 June 2002 / Published online: 14 August 2002

(C) Springer-Verlag 2002

B. Lee, K. Saitou ( $\square)$

University of Michigan,

Department of Mechanical Engineering,

2350 Hayward, Ann Arbor, MI 48109-2125, USA

E-mail: kazu@umich.edu

Fax: +1-734-6473170

We gratefully acknowledge financial support from the Engineering Research Center for Reconfigurable Machining Systems established by the National Science Foundation. manufacturability and in generating alternative designs of a product family. Because of the tight sharing of manufacturing resources among multiple product types, a slight change in a design feature can have a dramatic impact on the manufacturing cost. The generation of alternative designs and their quantitative manufacturability evaluation is therefore essential for the effective implementation of DFM for product family design.

In addition to variations in design, variations in production plans because of fluctuating market demand are another challenge to industry. In order to comply with this changing market demand, every decision regarding design and manufacturing should not only consider current market demand but also the long-term forecast of the production plan. Setting aside production plan changes, much effort has been made to reduce production cost by manipulating process planning, resource allocating and scheduling algorithms. Considering design's large impact on manufacturing cost, it is obvious that manufacturing cost would be reduced significantly if we make design decisions robust to production plan variations. Robustness to production plan variations represents, in this paper, achieving lower production cost with unchanged design of a part family throughout periods in the production plan.

In this work, a method is proposed to design product families that are robust to production plan variations, based on the quantitative evaluation of manufacturability, so that a designer can estimate the rough cost of production at the very early stage of the design process and obtain redesign suggestions. The proposed method aids the design of a family of machined products, i.e. part family, for a particular manufacturing system. It searches for a lower production cost by suggesting the best datum allocation for each member of a part family. Assuming that the geometry of a part is fixed by engineering requirements, datum definition has been chosen as the design variable, since the datum definition for geometric dimensions and tolerances is crucial to functional achievement and subsequent manufacturing processes of machined products. Suggesting the best datum allocation for each member of a part family can be justified when the cost to accommodate differently defined datum definitions is negligible compared to the reduction in the production cost for multiple production periods. Throughout this paper, a part family of a few members under mass production is considered.

Two examples are given to illustrate the effectiveness of the method. In each example, two slightly different prod- 
ucts are given along with production plans for certain periods of time. Alternative datum definitions are presented as a result of the proposed method.

\section{2}

\section{Related work}

Various methods to reduce the production cost of product families have been proposed. Whitney (1993) reported a case study of various strategic design methods to achieve flexibility against mixed models. Increasing the quantified commonality among a variety of assembled products has also been studied (Ishii et al. 1995; Kota et al. 2000). Adapting modularity and sharing platforms among products have also reduced design and manufacturing costs (Ulrich and Eppinger 1995; Fujita et al. 1998; GonzalezZugasti et al. 1998; Simpson et al. 1999; Nelson et al. 1999). However, the above work does not optimize the design of a product family to simultaneously minimize the facility cost and cycle time.

Grouping a wide variety of parts into part families has always been a research focus of studies related to group technology and cellular manufacturing systems. Nevertheless, few works in this area discuss redesign suggestions based on manufacturability feedback (Suresh and Kay 1998).

Hernandez et al. (1998) considered cycle time and market demand to design product families. Their method, however, has limits for direct application to machined products, where the analysis of function and geometrical tolerance of products are essential. Herrmann and Chincholkar (2000) have suggested the 'design for production' (DFP) method, where designers evaluate product designs by comparing their manufacturing requirements with an available production capacity and an estimated cycle time. Kusiak and $\mathrm{He}$ (1998) have suggested four 'design for agility' rules for product designs that are robust against the changes in the characteristics of production schedules. Although these methods suggest the reconsideration of specific design features, they cannot automatically generate redesign suggestions because of the lack of automated reasoning on the design features essential to the product function.

While numerous works have focused on automated manufacturability analysis (Gupta et al. 1997), a few of them addressed the automated generation of redesign suggestions. Das et al. $(1994,1996)$ proposed a methodology that proposes redesign suggestions of less setup time than original designs. By generating alternative machining features, the approach creates an extended feature set, combinations of which are evaluated in terms of setup time. Hayes and Sun (1995) utilized constraint networks (CN) and a knowledge-based system to analyze a machined product. They developed a system that generates design modification in terms of tolerance and datum selections with minimal processing time, including setups. The method was successively applied for shape-changing redesign suggestions (Hayes 1996). Although these methods generate redesign suggestions by evaluating multiple choices of feature recognition and process planning, they were not extended to application for the production of part families. Figure 1 summarizes the past work done on redesign suggestions for machined parts.

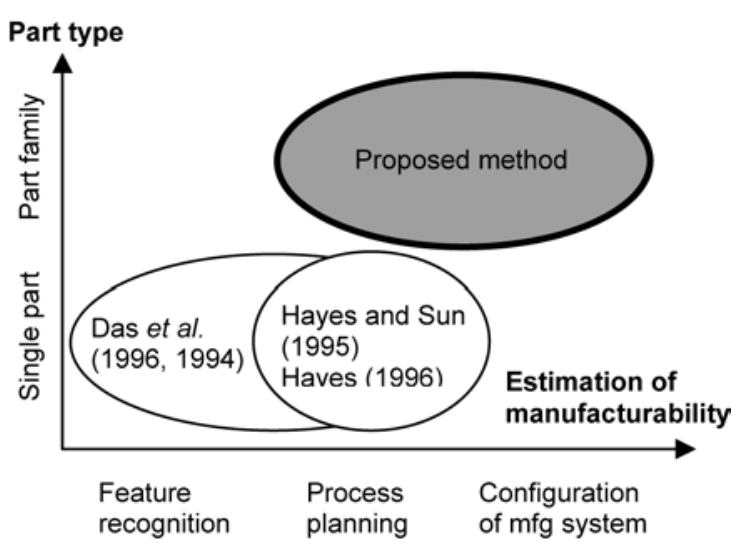

Fig. 1. A map of the past work on redesign suggestions for machined parts

\section{3}

\section{Approach}

When two or more products are being produced within a manufacturing system, process plans of those products with minimal setup and processing time do not necessarily result in minimal cycle time and manufacturing system configuration. For this reason, unlike most DFM methods, the proposed framework quantitatively evaluates the manufacturability of the part designs based on the facility cost and cycle time for production, which are estimated by the discrete event simulation of part production during multiple periods. Given initial designs of parts in a family and variations of production plan (volume ratio of the product mix), the method generates redesign suggestions realizing lower production and facility costs based on the following four steps (Fig. 2). As shown in Fig. 2, each step incorporates several substeps over a span of a decision tree involved in design and manufacturing. This simplified decision tree consists of several steps in which multiple choices are typically available from one step to the next. Eventually, the best decisions throughout the tree will be identified to provide redesign suggestions to the initial designs. Each step will be fully described in the following subsections.

1. Feature recognition: transform the initial designs into the constraint networks $(\mathrm{CN})$ of tolerance $s$ among machining features by extracting the precedence relationship among machining features within the nodes in the graphical representation of the initial designs. While in general a design can be transformed to multiple CN (Das et al. 1994, 1996), we assume a design is transformed to a unique CN. This is because we concentrate on subsequent steps, which are essential for evaluating the manufacturability of a part family, to keep the size of problem manageable.

2. Generation of alternative designs: generate alternative designs (i.e. $\mathrm{CN}$ ) for the part family, which are candidates for redesign suggestion. These alternative designs satisfy the functional requirement of the original designs and the generic tolerance rules. In this paper, the functional requirement for a part is referred to as the 


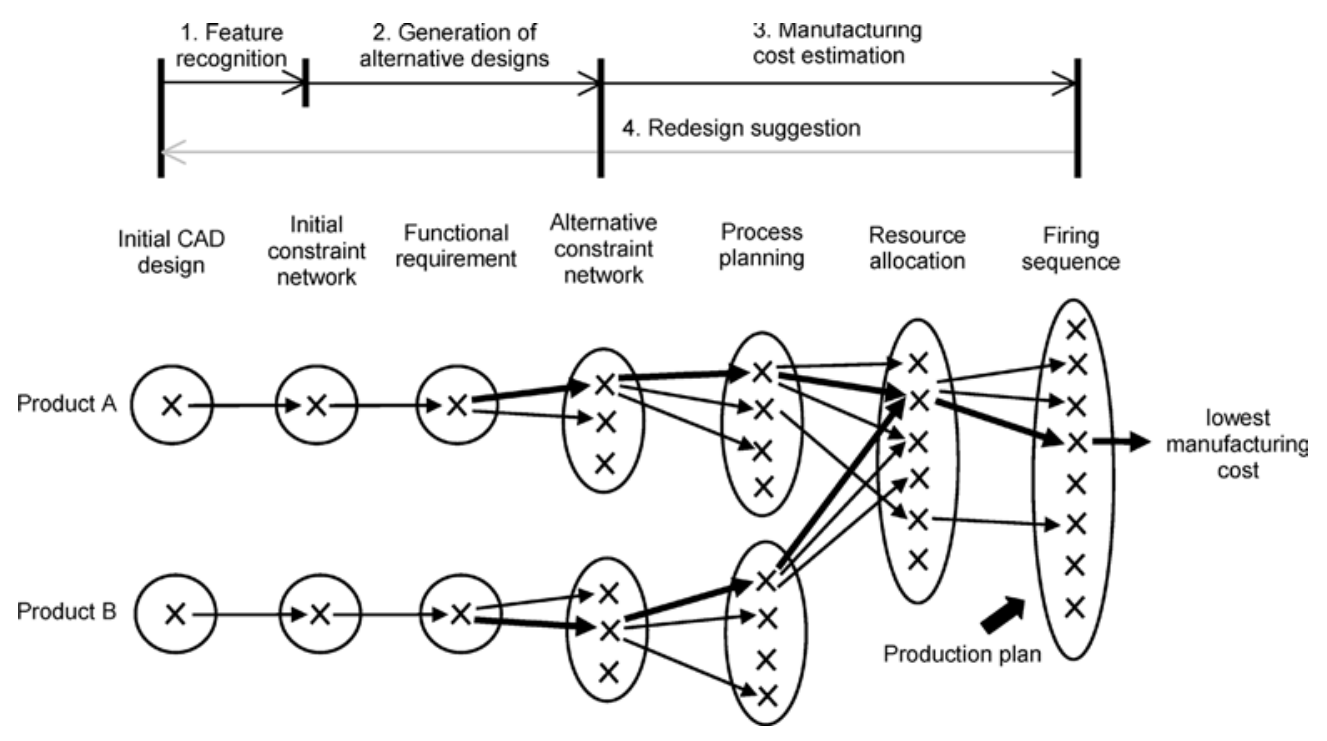

\begin{abstract}
Fig. 2. Computational framework for redesign suggestion system. The lower part of the figure shows the 'design and manufacturing' decision tree for a part family of two members
\end{abstract}

core datum relationship, which is indispensable for the part to satisfy its role by itself or in the assembly to which it belongs.

3. Manufacturing cost estimation: estimate the manufacturing cost for each set of $\mathrm{CN}$ of the part family generated in step 2. Based on the process precedence imposed by the alternative $\mathrm{CN}$, estimate the lowest production and facility cost of the part family under a given production plan by optimizing process plans, resource allocation (mapping from manufacturing features to machine stations) and firing sequence (the order of product types to be fed into the manufacturing system). A simple discreteevent model of manufacturing systems is used to evaluate the production cycle time.

4. Redesign suggestion: find a new $\mathrm{CN}$ that gives the lowest manufacturing cost estimation obtained in step 3 for the given production plan variations. Update initial designs with the new $\mathrm{CN}$.

\section{1}

\section{Feature recognition}

\subsection{1}

\section{Graphical representation of product information}

A compact, graph-based representation of product geometry and tolerances has been utilized to evaluate and manipulate product designs. The graph consists of a modified attributed adjacency graph (AAG, proposed by Joshi and Chang 1988) representing the geometry information, overlapped with the directed edges representing the tolerance and datum relationships among geometry primitives.

A node represents a geometry primitive such as a cylindrical face, and an undirected edge between nodes represents a physical edge that those nodes share. Upon the geometry information, tolerance and datum information is added in the form of directed edges with tolerance information, because the dimension for a geometry feature to be processed is defined from a reference feature with a proper tolerance level. The graph described above can be defined as a sextuple:
$G=(V, U, D, A v, A u, A d)$,

where $V$ is the set of nodes, $U$ is the set of undirected edges, $D$ is the set of directed edges, $A v=\{P F, C F, \ldots\}$ is the set of attributes to node set $V, A u=\left\{c+, c_{-}, s+, s^{-}, \ldots\right\}$ is the set of attributes to undirected edge set $U$ and $A d=\{x 2$, $y 2, z 2, p 2, \ldots\}$ is the set of attributes to directed edge set $D$.

Every node $v$ in $V$ is assigned an attribute $a v$ in $A v$, such as $a v=$ ' $P F$ ' for a planar face. Every undirected edge $u$ in $U$ is assigned an attribute $a u$ in $A u$, such as $a u=$ ' $c+$ ' for a cylindrical edge that forms a convex angle and $a u=$ ' $s-$ ' for a straight edge that forms a concave angle. Most of these notations including node indices have been borrowed from $\mathrm{Fu}$ and dePennington (1994). Likewise, every directed edge $d$ in $D$ is assigned an attribute $a d$ in $A d$, such as $a d=' x 2$ ' for the positional tolerance with level of the second decimal point and $a d=$ ' $p 2$ ' for perpendicularity with the same level of tolerance.

Figure 3 shows a simple L-shaped bracket and its graphical representation. For example, in Fig. 3a the vertical face defined as datum ' $B$ ' shares a straight convex edge with the horizontal face defined as datum ' $A$ '. This relationship is represented in Fig. $3 b$, where PF1 (vertical face) and PF6 (horizontal face) are connected with an undirected edge with attribute is ' $s+$ ' (straight convex). Also, the perpendicularity between those faces in Fig. 3a is represented as a directed edge of ' $p 2$ ' attribute in Fig. $3 b$. In this example, the constraints with tolerance looser than the second decimal point and the reference dimensions are ignored, assuming they can be met from the stock material before machining operations. For the same reason, faces used as datums are recognized as planar faces that require milling operations, even if they do not have obvious volumetric machining features. The number in a node after the attribute is the node index.

\subsection{2}

\section{Constraint network after feature recognition}

From the geometry and tolerance information a feature recognition method transforms an AAG representation of the product into a $\mathrm{CN}$ defined among manufacturing 


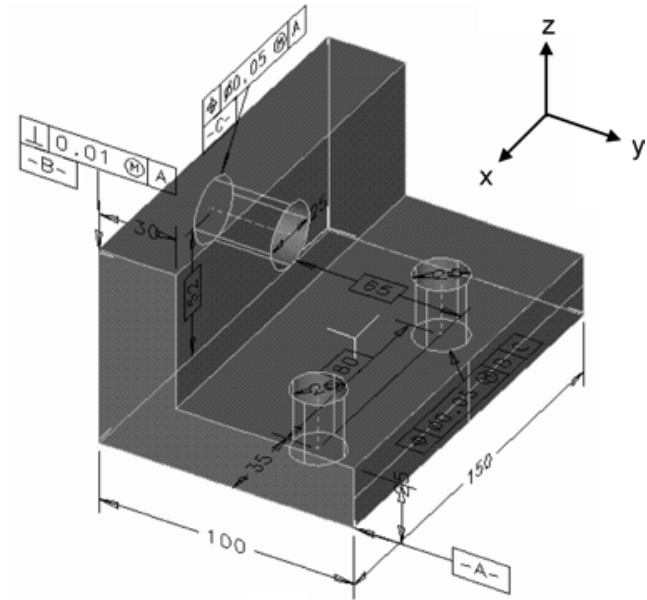

(a)

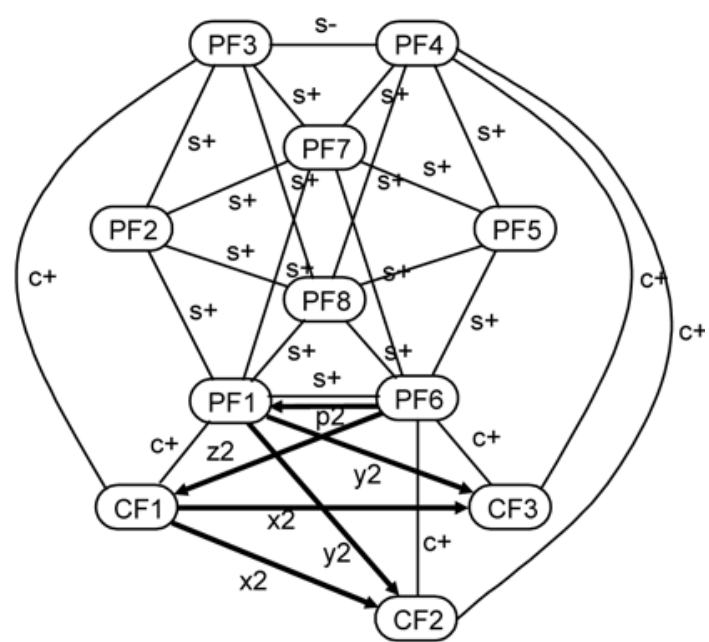

(b)

Fig. 3. a Solid model of a part and $\mathbf{b}$ its graphical representation

features. This constraint network provides the precedence relationship among manufacturing features for the process planning. The constraint network can be defined as a fourtuple:

$C N=(F, T, A f, A t)$,

where $F$ is the set of nodes, $T$ is the set of directed edges, $A f$ is the set of attributes to node $F$ and $A t$ is the set of attributes to directed edge $T$.

A node represents a manufacturing feature, and a directed edge represents the tolerance and datum information inherited from the graph $G$. Each node has attributes such as the volume of metal to be removed, the orientation of tool approach and the type of feature for the process planning.

Figure 4 shows a constraint network after feature recognition is applied to the graph shown in Fig. 3b. Note that cylindrical face ' $C F 1$ ' on the vertical plane (see Fig. 3) is recognized as a through hole ('TH1') and a set of parallel holes ' $C F 2$ ' and ' $C F 3$ ' on the lower place is recognized as parallel holes (' $\mathrm{PH1}$ ') to simplify the problem, assuming a double-spindle drilling machine is available.

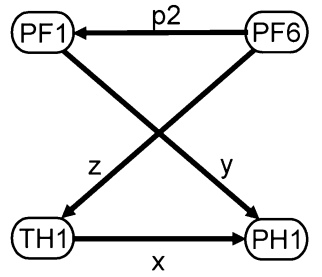

Fig. 4. Constraint network among manufacturing features obtained from the AAG of the product in Fig. 3

\section{2}

\section{Generation of alternative designs}

This section describes generating alternative datum selections that give better cycle times, based on the framework illustrated in Fig. 2. The functional requirements of machined parts are often achieved by the tolerance relationship of features. For example, if two products are assembled using bracket holes shown in Fig. 3, and if their relative location in the $x$-direction is important, the relative location of the two bracket holes must be kept precise, which necessarily means tight tolerance among the holes. When the initial designs are provided, we assume that all tolerance relationships are indispensable for functional requirements. Alternative datum selections are searched for among the choices that conform to the functional requirements of the products. The system searches for such alternative datums based on following sequence (Fig. 5).

1. The functional requirements (Fig. $5 \mathrm{~b}$ ) are extracted from the initial CN (Fig. 5a), where a dashed line means there exists a path with the designated attribute between the two nodes connected by the line, or there exists another node other than the two nodes from which paths to the two nodes exist.

2. The system randomly selects only one manufacturing feature (node in $\mathrm{CN}$ ) for one type of geometry tolerance (Fig. 5c). For example, in a prismatic part the positional tolerances in the $x$-direction may have one planar face

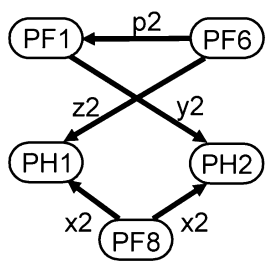

(a) initial $\mathrm{CN}$

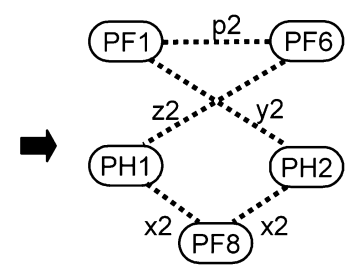

(b) functional requirement

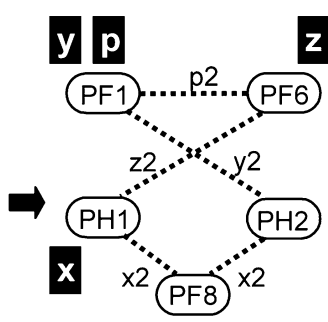

(c) select datums

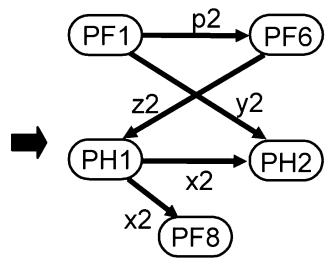

(d) recompose $\mathrm{CN}$
Fig. 5a-d. Steps to generate alternative designs 
as the only datum whose normal vector is parallel to the $x$-axis. This rule is reasonable and conventional for typical parts if they are not highly complicated.

3. The system recomposes the $\mathrm{CN}$ to be consistent with the functional requirements and the selected datums.

In addition, the following three rules are strictly kept while manipulating the $\mathrm{CN}$ in order to guarantee that all features within the $\mathrm{CN}$ are well constrained in terms of tolerance. Alternative designs generated according to the procedure described above satisfy the first two of three rules automatically; the designs that do not satisfy the third rule are discarded.

1. An isolated node, that is, an unconstrained feature, should not exist in a $\mathrm{CN}$, because it is assumed that tolerances looser than some degree are met by the raw material. Nodes with self-referenced tolerance such as flatness are exceptions to the rules.

2. A node is not allowed to have more than one incoming edge with the same type of tolerance. Otherwise, the feature is over-constrained or one of the incoming edges with looser degree is redundant.

3. A loop is not allowed in a CN. When a loop is formed, every node within the loop has at least one incoming edge coming from another node within the same loop, hence any edge cannot be processed unless one of them has been processed. Loops that consist of only one node are allowed and processed as self-referenced tolerances.

The rules discussed above can be found in a slightly different form in Tsai and Cutkosky (1997), where more information about the representation and reasoning of geometrical tolerances is provided in depth.

\section{3}

\section{Manufacturing cost estimation}

\subsection{1}

\section{Modeling of process planning, manufacturing system configuration and firing sequence}

For a given set of two or more product designs and production plans for a time period, the system searches for the best process plans, manufacturing system configurations and firing sequence to obtain minimal cycle time and facility cost. The process plan specifies a sequence of manufacturing features that satisfies the precedence condition represented in the constraint network. In order to build manufacturing system configurations, we also need to decide the allocation of manufacturing features in the process plan to machine stations in the manufacturing system configuration, which can be represented as a mapping from manufacturing features to machine stations.

Let $F$ be a set of manufacturing features and $S$ that of machines. A process plan can be represented as a sequence p, which includes every node $f \subset F$ as its components without duplication. Resource allocation can be represented by Corr: $F \mapsto S$. It is assumed that a mapping Corr has following characteristics:

Corr maps a manufacturing feature $f \subset F$ to a machine $s \subset S$ with the matching attribute. For example, it will assign a through hole to a drilling machine but not to a milling machine.

Corr maps a manufacturing feature $f \subset F$ to a unique machine $s \subset S$. In other words, a process is assigned to a unique machine. This implies that a product is allowed to pass the manufacturing system via a unique route, prohibiting 'loops' in the transfer line.

When the process plan and the machine allocation are decided, we can build a manufacturing system configuration by linking machines and assigning attributes to them. The manufacturing system configuration is defined as a four-tuple:

$\operatorname{Config}(\mathbf{p}$, Corr $)=(S, E, A s, A e)$,

where $S$ is the set of nodes, $E$ is the set of directed edges, $A s$ is the set of attributes to nodes in $S$ and $A e$ is the set of attributes to directed edges in $E$.

A node in $S$ represents a machine station, and a directed edge in $E$ represents a transfer line. An attribute of a node represents the type of machine such as face milling or drilling. An attribute of a directed edge represents the product type that can be accepted by the transfer line represented by the directed edge. Since it is assumed that automated transfer lines are installed between machines and no intermediate buffer is allowed for the simplicity of problem, a product is allowed to visit a machine only once during the production (i.e. no 'cyclic' production) to avoid system deadlock.

In addition, since there is no buffer assumed between machine stations, no scheduling rule is necessary except for the firing sequence at the start buffer of the manufacturing system. The firing sequence is a finite sequence of the product types that are waiting to enter a machine. For instance, if the production plan, which is the volume ratio of two products $A$ and $B$, is $1: 3$ and the cycle of sequence is 8 , the firing sequence could be $A B B B B A B B$. The firing sequence can be denoted by sequence $s$, whose components are the elements of the attribute set $A e$ of Config.

\subsection{2}

\section{Discrete event simulation for cycle time estimation}

Once the process planning, manufacturing system configuration and firing sequence are decided, a discrete event simulation is run to estimate the average cycle time. The average cycle time means the average time span spent to obtain a completed product after obtaining the previous one.

$\mathrm{CN}$ of two products and the corresponding process plans and manufacturing system configurations are depicted in Figs. $6 \mathrm{a}, 6 \mathrm{~b}$ and $6 \mathrm{c}$, respectively. In Fig. $6 \mathrm{c}$, the start buffer will fire a raw material of product $A$ to a empty node or machine station, which is in this case the node marked ' $F M$ ', where the outgoing edge with attribute ' $A$ ' is pointing. The amount of time for a manufacturing feature to spend on its corresponding machine is decided by the volume of material to be removed divided by the material removal rate of the machine, plus setup time if orientation of tool approach is different from that of the previous feature (Huang et al. 1997). As the computed time passes, the product $A$ is ready 


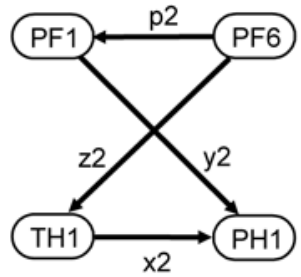

(a)

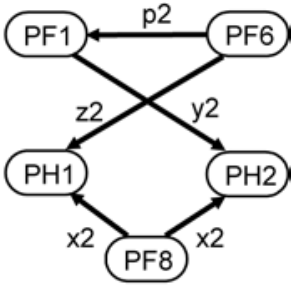

(b)

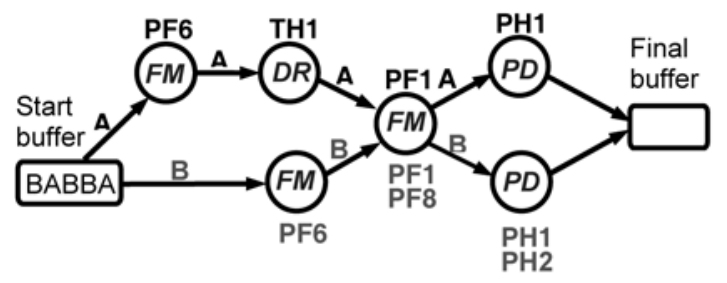

(c)

Fig. 6. a Constraint network of product $A ; \mathbf{b}$ constraint network of product $B$; c corresponding manufacturing system configuration and firing sequence that produces products $A$ and $B$ simultaneously

to leave to the next node. If the next node is empty, the product $A$ is delivered to the next station ' $D R$ ' via the edge whose attribute a $e={ }^{\prime} A$ '. The discrete event simulation algorithm is based on colored Petri nets (Alla et al. 1985) implemented for our previous work on manufacturing system optimization (Saitou and Malpathak 1999).

Time is measured from when a product arrives at the final buffer for the first time to when the number of products contained in the final buffer reaches the specified sequence cycle. Then the measured time is divided by the sequence cycle to obtain the average cycle time $t_{c}$.

\subsection{3}

\section{Simultaneous optimization of process planning, manufacturing system configuration and firing sequence}

For each set of CN generated in Sect. 3.3.2 and a production plan, the lowest manufacturing cost is estimated by simultaneously optimizing the process plans, manufacturing system configuration and firing sequence. The production plan for a given period of time is specified as the fraction of each type of products and the amount of time $\tau$ for which the production plan is kept. The time $\tau$ is assumed to be on the order of a few years; hence the cost for reconfiguration of the manufacturing system can be ignored. Let $n$ be the number of types of products and the fraction be $\alpha_{i}$, where $0 \leq \alpha_{1} \leq N$ for $i=1,2, \ldots, n$ and $\sum_{i=1}^{n} \alpha_{i}=N$ for some constant $N$, or collectively be a $n$-dimensional vector $\boldsymbol{a}$. Therefore, a production plan can be defined as a function of time span $\tau$ and the fraction vector $\boldsymbol{a}$, which we shall call $\rho(\tau, \boldsymbol{a})$.

Our objective is to minimize facility cost while achieving efficient production. Facility cost is assumed to include the running cost of machines and the interest of investment and depreciation, and to be simply dependent on the number of machines, i.e. the number of nodes $|S|$ in Config. Efficiency of production is assumed to be measured simply by the production cycle time. Hence, the overall cost for production of a product family for a production plan can be estimated as the product of amount of time period $\tau$ for a production plan and the summation of the number of machines $|S|$ and the average cycle time $t_{\mathrm{c}}$ weighted by a set of constants.

\section{4 Redesign suggestion robust to variations of production plan}

This is the final step to find and suggest alternative designs with reduced cost. Assuming that the forecasts on production plans for multiple periods of time of interest are available as a sequence of $m$ production plans $\rho\left(\tau_{j}, \boldsymbol{a}_{j}\right)$ for $j=1,2, \ldots, m$, we search for a set of $\mathrm{CN}$ of $n$ products that minimizes gross cost throughout the time periods of interest. Product designs remain the same for $m$ periods, while the manufacturing system configuration and firing sequence are varied to provide the lowest manufacturing cost for each period. Then the manufacturing cost for each period weighted by the amount of each time period $\tau$ is summed to obtain the gross cost.

In summary, the whole procedure, including routines described in Sect. 3.3.3 and this section to find the optimal datum selections that are robust to the variations in the production plans, can be stated as follows:

given: initial constraint network $C N_{i 0}$,

production plan variations $\rho\left(\tau_{j} a_{j}\right)$ and

lengths of firing sequence $\left|s_{j}\right|$,

find : constraint networks $C N_{i}$,

process plans $\mathbf{p}_{i j}$,

mappings Corr $_{i j}$,

mfg system configurations Config $_{j}$ and

firing sequences $s_{j}$

satisfying :

functional requirements,

tolerance rules

that minimize :

grosscost $=\sum_{j=1}^{m} \tau_{j} \times\left(w_{p} \times t_{c j}+w_{f} \times \mid\right.$ Config $\left._{j} \mid\right)$,

where $w_{p}$ and $w_{f}$ are weights for $i=1,2, \ldots, n$

and for $j=1,2, \ldots, m$.

Redesigns are suggested based on the best set of $\mathrm{CN}$ for $n$ products obtained from this procedure. Tolerance relationships of original designs are then updated with these suggested $\mathrm{CN}$.

\section{4}

\section{Software implementation}

Because the problem is highly complex, a multistage optimization scheme utilizing a genetic algorithm (Holland 1975; Goldberg 1989) is adopted (Fig. 7). At the top level of the scheme, candidate $\mathrm{CN}$ are generated at the first-stage 


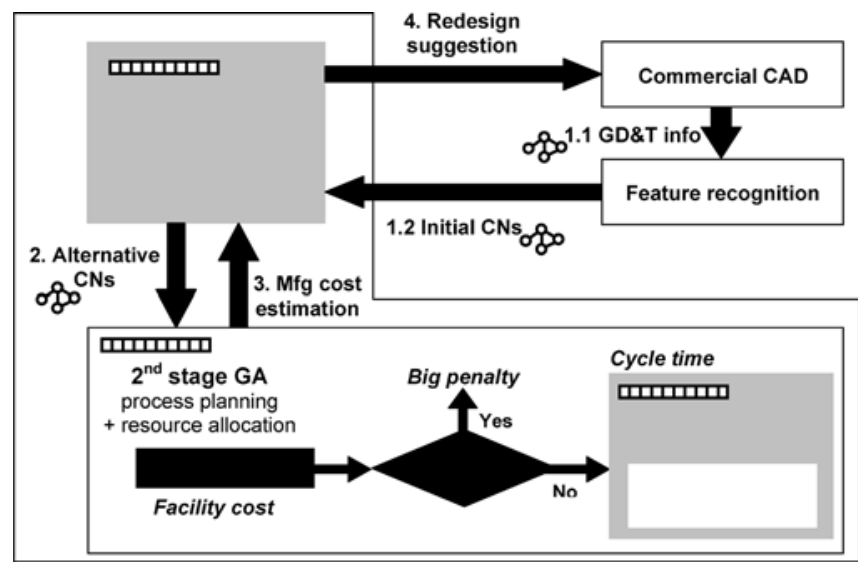

Fig. 7. Software implementation. Numbers indicate steps described in Sect. 3

genetic algorithm (GA) according to functional requirement and tolerance rules as described in Sect. 3.2. Then they are passed into the second-stage GA, where the routine described in Sect. 3.3.3 is initiated to estimate the lowest manufacturing cost for the set of $\mathrm{CN}$. At this stage, if no loop is detected, candidate pairs of a process planning and a manufacturing system configuration are passed into the third-stage GA, where the firing sequence with minimum average cycle time is decided through the discrete event simulation system. For each set of alternative $\mathrm{CN}$, the second-stage GA and below is repeated $m$ times to obtain the manufacturing cost for each volume ratio specified in the production plan variations. When this iteration is complete for all alternative $\mathrm{CN}$, the system has estimated gross manufacturing cost for every set of alternative CN. Then the first-stage GA decides the best set of $\mathrm{CN}$ with which to update original designs. Automatic feature recognition and updating of original designs are not incorporated into system and remain as future work.

Since this scheme excludes infeasible solutions at each step without running the whole procedure for every candidate solution, it allows a faster evaluation. However, it should be noted that the generated solution is not guaranteed optimal due to the multistage optimization formulation and the stochastic nature of the genetic algorithm. The computer software is written in $\mathrm{C}++$ with the intensive use of data types and algorithms of LEDA (Mehlhorn and Näher 1999) developed at Max Planck Institut für Informatik, (Saarbrücken, Germany). Also, the genetic algorithm within the software is implemented using GAlib ${ }^{1}$. developed at MIT CADLAB.

\section{5}

\section{Examples}

In this section, two simple case studies are provided to show the effectiveness of our method with simulation results. In each case study production plan variation for three periods $(m=3)$ is considered and a set of redesign suggestions for two products $(n=2)$ are suggested at the end of the results.

\footnotetext{
${ }^{1}$ Documentation of this library is available at http://lancet.mit.edu/ga/
}
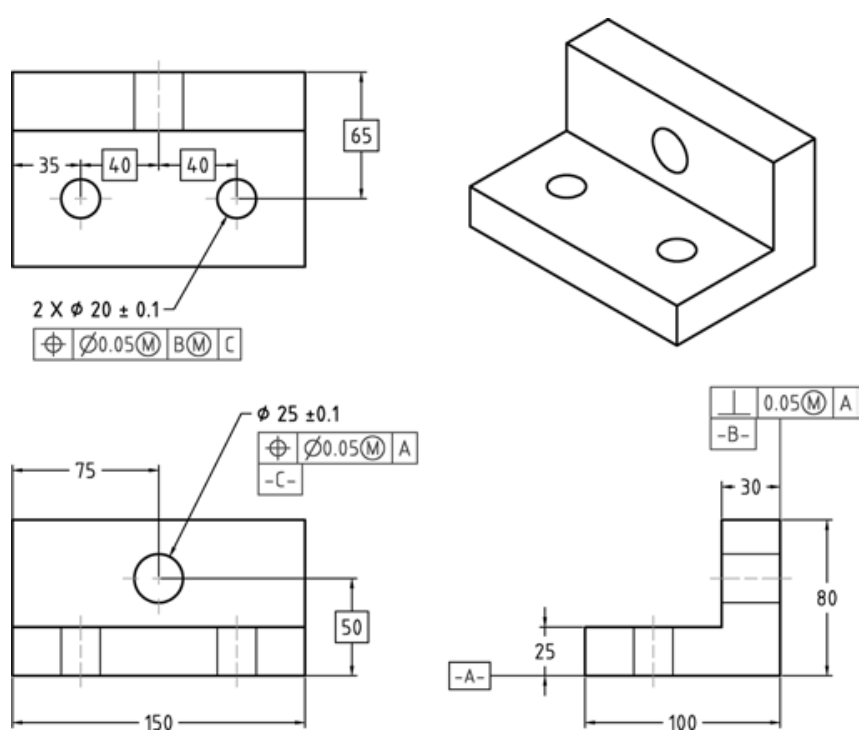

Fig. 8. Original design of product $A$ in example 1
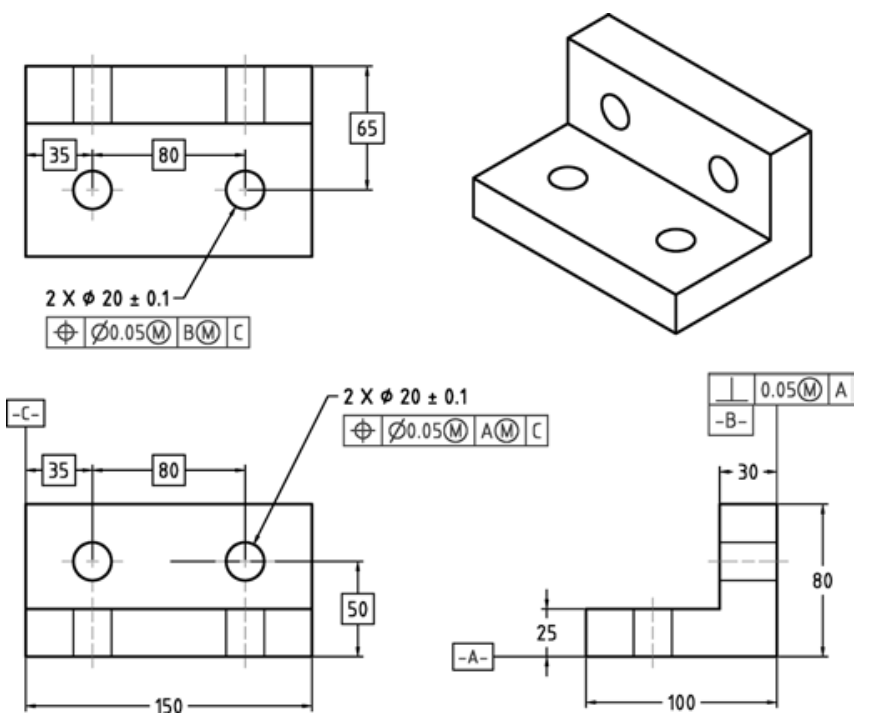

Fig. 9. Original design of product $B$ in example 1

\section{1}

\section{Example 1: L-shaped brackets}

The initial CAD model and graphical representation for two products $A$ and $B$ are depicted in Figs. 8 and 9, respectively. Also, $C N$ of products $A$ and $B$ can be found in Figs. $6 \mathrm{a}$ and $6 \mathrm{~b}$, respectively, in which the datum definitions for the two products are quite similar except for the positional tolerance of two holes in the $x$-direction. This resemblance comes from a common design practice, that is, when more than two similar designs are launched together or when one product is designed after the other, their datum definitions are similar to reduce cost for production plan changes and to utilize existing fixtures.

All nodes and attributes of edges (type of tolerance) are assumed to be essential to fulfill functional requirements. Then the functional requirements for the two products can be described as two graphs with dashed edges (Fig. 10), where a dashed line means there exists a path between the 
two nodes it connects, or there exists another node other than the two nodes from which paths to the two nodes exist, as described in Sect. 3.2.

Production plans for the three periods and the length of the sequence cycle are given as follows: $\rho\left(\tau_{1}, \boldsymbol{a}_{1}\right)=\rho(1,(9,1))$, $\rho\left(\tau_{2}, \boldsymbol{a}_{2}\right)=\rho(3,(5,5)), \rho\left(\tau_{3}, \boldsymbol{a}_{3}\right)=\rho(3,(2,8))$ and $\left|\boldsymbol{s}_{j}\right|=10$ for all $j$. Figure 11 diagrams the production plan variations. The weight for the cycle time and the weight for the number of machines are set to $w_{p}=10$ and $w_{f}=65$, respectively, so that the number of machines of optimum solutions does not reach its lower or upper bound. The material volume to be removed for each manufacturing feature in products $A$ and $B$ is listed in Table 1 . We use $P F$ to represent a planar face, $\mathrm{TH}$ to represent a through-hole and $\mathrm{PH}$ to represent a pair of parallel holes. The material removal rate is given as 2,4 and 7 for the face mill, drill and parallel drills, respectively.

A set of optimized CN for two products, Config for three periods and the accompanying six process plans obtained after running the routine described in Eqs. (4), (5), (6) and (7) are presented in Figs. 12 and 13, respectively. Two black edges in the $\mathrm{CN}$ for product $A$ (Fig. 12a) indicate that they are reversed as a result of the search for minimum cost. No modification of product $B$ has been suggested (every edge in the $\mathrm{CN}$ for product $B$ shown in Fig. $12 \mathrm{~b}$ is colored gray). Figure 14 shows the updated design with the modified $\mathrm{CN}$ of product $A$. The estimated costs are also given in Table 2 and are compared with the results for the original designs with same production plan variations. Although the total processing time and the number of setups has not decreased in this case (Table 3), reversing the two datum definitions has achieved a cost reduction of $3.7 \%$ (see gross cost in Table 2).

Figure 13 shows process plans and manufacturing system configurations for three periods and modified designs. For the first period of the production plan when the volume ratio between $A$ and $B$ is 9:1, product $A$ is routed
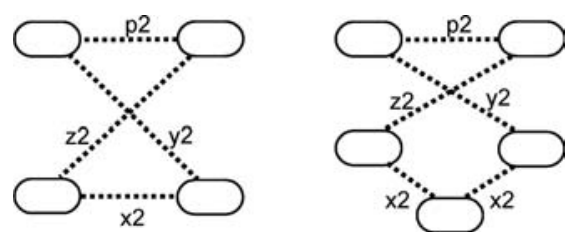

Fig. 10. Representation of functional requirement for example 1

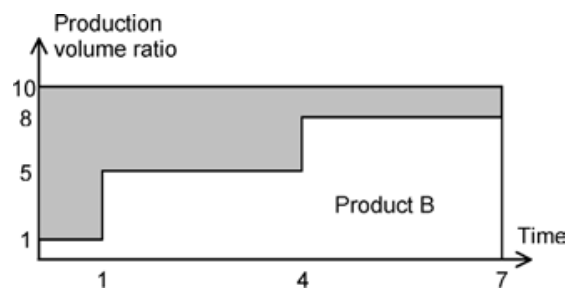

Fig. 11. Production plan variations for example 1

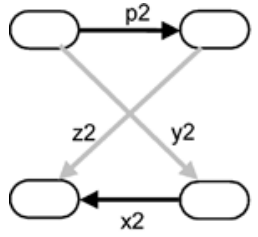

(a) $-C N_{1}$

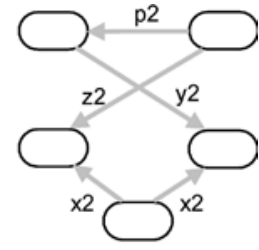

(b) $-\mathrm{CN}_{2}$
Fig. 12a, b. Redesign suggestion of constraint networks of product $A$ and $B$ in example 1. Black edges represent reversed edges from the original $\mathrm{CN}$

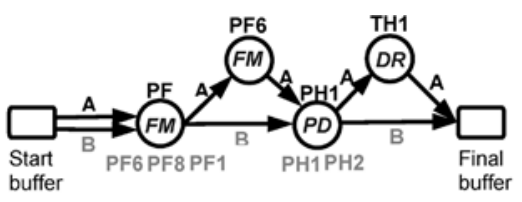

(a) - Config ${ }_{1}, \mathbf{p}_{11}, \mathbf{p}_{21}$, Corr $_{11}, \operatorname{Corr}_{21}$ for $\rho\left(\tau_{1}, \mathbf{a}_{1}\right)$

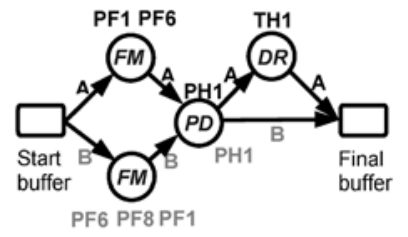

(b) - Config $2, \mathbf{p}_{12}, \mathbf{p}_{22}$, Corr $_{12}$, Corr $_{22}$ for $\rho\left(\tau_{2}, \mathbf{a}_{2}\right)$

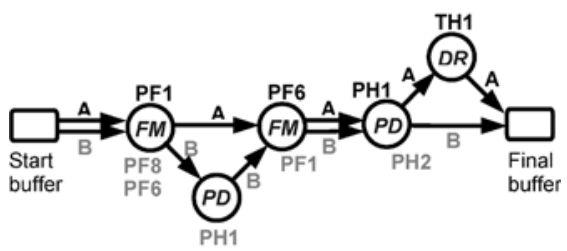

(c) - Config ${ }_{3}, \mathbf{p}_{13}, \mathbf{p}_{23}, \operatorname{Corr}_{13}, \operatorname{Corr}_{23}$ for $\rho\left(\tau_{3}, \mathbf{a}_{3}\right)$

Fig. 13a-c. Process plans and manufacturing system configuration corresponding to three periods of production plan (Fig. 11) and the redesign suggestions (Fig. 12)

through four machines, while product $B$ uses two machines. For the second period when the volume ratio is 5:5, the number of machines that each product uses changes to 3 versus 2 . And for the third period, it is 4 versus 4 . Although product $A$ uses four machines for this period, the number of machines only for product $A$ is 1 . From this observation, it is obvious that a higher-volume product dominates the configuration of the manufacturing system.

To show that redesign suggestions can differ according to production plan variations, we generated three sets of optimal redesign suggestions for each volume ratio in the production plans shown in Fig. 11. Each pair of CN presented in Fig. 15 is a better solution than the robust solution depicted in Fig. 12, as far as each production plan goes. For example, if production plan of 5:5 (period 2) is kept throughout the time periods, the solution in Fig. 15b
Table 1. Material volume to be removed for manufacturing features in example 1

\begin{tabular}{lllllll}
\hline Product $A$ & Feature & PF1 & PF6 & TH1 & PH1 & - \\
& Volume & 20 & 22 & 50 & 90 & - \\
Product $B$ & Feature & PF8 & PF1 & PF6 & PH1 & PH2 \\
& Volume & 10 & 22 & 20 & 90 & 90
\end{tabular}



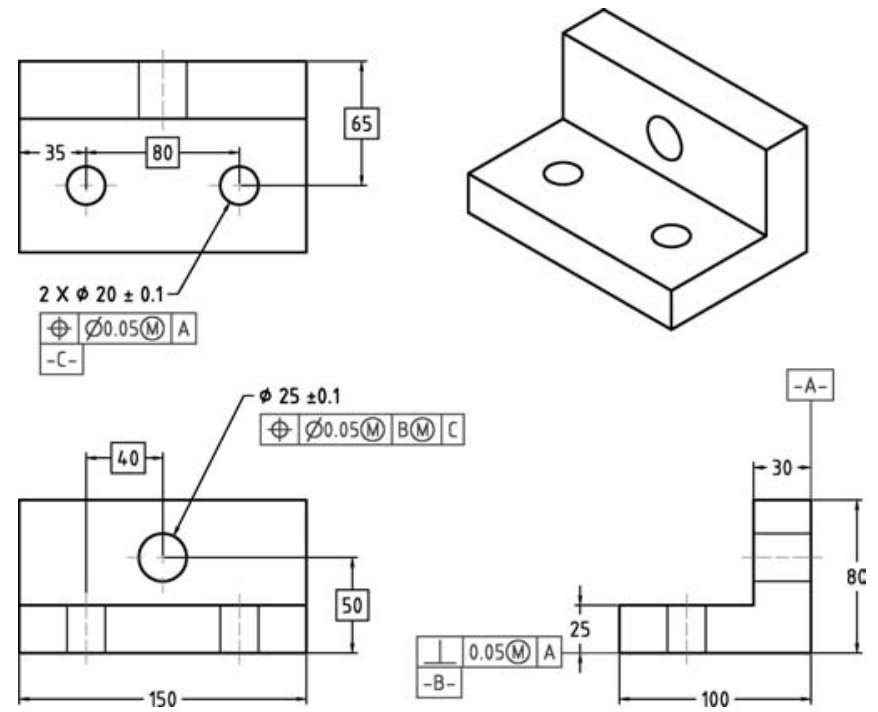

Fig. 14. Updated design of product $A$ in example 1

will cost less than the solution. Since example 1 is very simple and is limited in terms of choices of datum sources, all process plans of Fig. 15 and those of the robust solution converged to production plans with the minimum number of setups, which is crucial to shorter cycle times. In fact, the solution for period 1 is identical to the robust solution, which shows that the design space is very limited and there are not many candidates with the minimum number of setups.

\section{2}

\section{Example 2: oscillator housings}

More complicated turned parts are studied in example 2. A CAD model and graphical representation for two products $A$ and $B$ are depicted in Figs. 16 and 17, respectively. Also, $\mathrm{CN}$ of products $A$ and $B$ can be found in Fig. 18. Product $A$ is directly adopted from Madsen et al. (1991), and product $B$ is modified slightly from product $A$. Functional requirements for the two product can be described as two graphs with dashed edges in Fig. 19, in the same way as in example 1.

Production plans for three periods and the length of the sequence cycle are given as follows: $\rho\left(\tau_{1}, \boldsymbol{a}_{1}\right)=\rho(2,(8,2))$, $\rho\left(\tau_{2}, \boldsymbol{a}_{2}\right)=\rho(3,(5,5)), \rho\left(\tau_{3}, \boldsymbol{a}_{3}\right)=\rho(3,(2,8))$ and $\left|\boldsymbol{s}_{j}\right|=10$.

Figure 20 shows the production plan variations diagram-
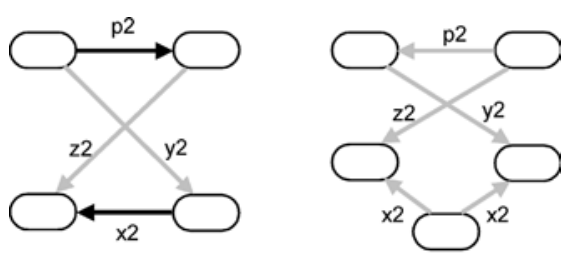

(a) - $C N_{1}$ and $C N_{2}$ for period 1
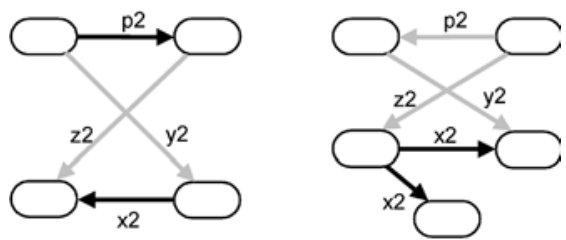

(b) - $C N_{1}$ and $C N_{2}$ for period 2
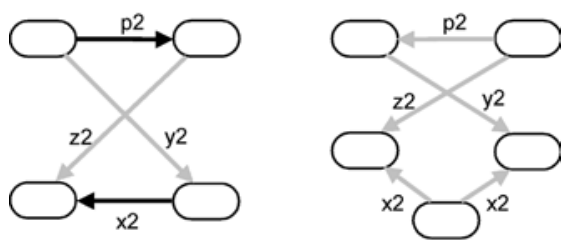

(c) - $C N_{1}$ and $C N_{2}$ for period 3

Fig. 15a-c. Each pair of constraint networks of products $A$ and $B$ is optimized for each volume ratio shown in Fig. 11. For each volume ratio, these $\mathrm{CN}$ give a lower cost than those in Fig. 12 optimized throughout the production periods

matically. The weight for the cycle time $w_{p}=20$, and the weight for the number of machines $w_{f}=55$. The material volume to be removed for each manufacturing feature in product $A$ is presented in Table 4 . Table 5 gives the available types of machines and their material removal rates (MRR) and matching manufacturing feature types. For turning machines, the time for tool changes is assumed to be negligible.

A set of optimized CN for two products is presented in Fig. 21, and Config for three periods and the accompanying six process plans obtained after running the routine described in Eqs. (4), (5), (6) and (7) are presented in Fig. 22. Figures 23 and 24 show the updated designs with modified $\mathrm{CN}$ of products $A$ and $B$, respectively. More design changes have been made than in example 1 because increased complexity provides the system with a wider range of choices for alternative
Table 2. Cost comparison between the original and modified designs - example 1

Table 3. The number of setups in the process plans from the redesign suggestion

\begin{tabular}{lllll}
\hline & \multicolumn{2}{l}{ Overall cost for period $j$} & \multicolumn{2}{l}{$\begin{array}{l}\text { Gross } \\
\text { cost }\end{array}$} \\
\cline { 2 - 3 } & $j=1$ & $j=2$ & $j=3$ & \\
\hline Original designs & 501.111 & 526.111 & 566.111 & 3777.777 \\
Modified designs & 493.333 & 497.778 & 549.444 & 3635.000 \\
\hline
\end{tabular}

\begin{tabular}{|c|c|c|c|c|c|c|c|}
\hline \multirow{2}{*}{ Part } & \multirow{2}{*}{$\begin{array}{l}\text { Process plans } \\
\mathbf{p}_{11}, \mathbf{p}_{12}, \mathbf{p}_{13}\end{array}$} & \multicolumn{5}{|c|}{ Orientations of machining features in sequence } & \multirow{2}{*}{$\begin{array}{l}\text { No. of } \\
\text { setups }\end{array}$} \\
\hline & & $-y$ & $-z$ & $z$ & $y$ & - & \\
\hline \multirow[t]{2}{*}{ B } & $\mathbf{p}_{21}, \mathbf{p}_{22}$ & $-z$ & $-x$ & $-y$ & $y$ & $z$ & 4 \\
\hline & $\mathbf{p}_{23}$ & $-x$ & $-z$ & $y$ & $-y$ & $z$ & 4 \\
\hline
\end{tabular}




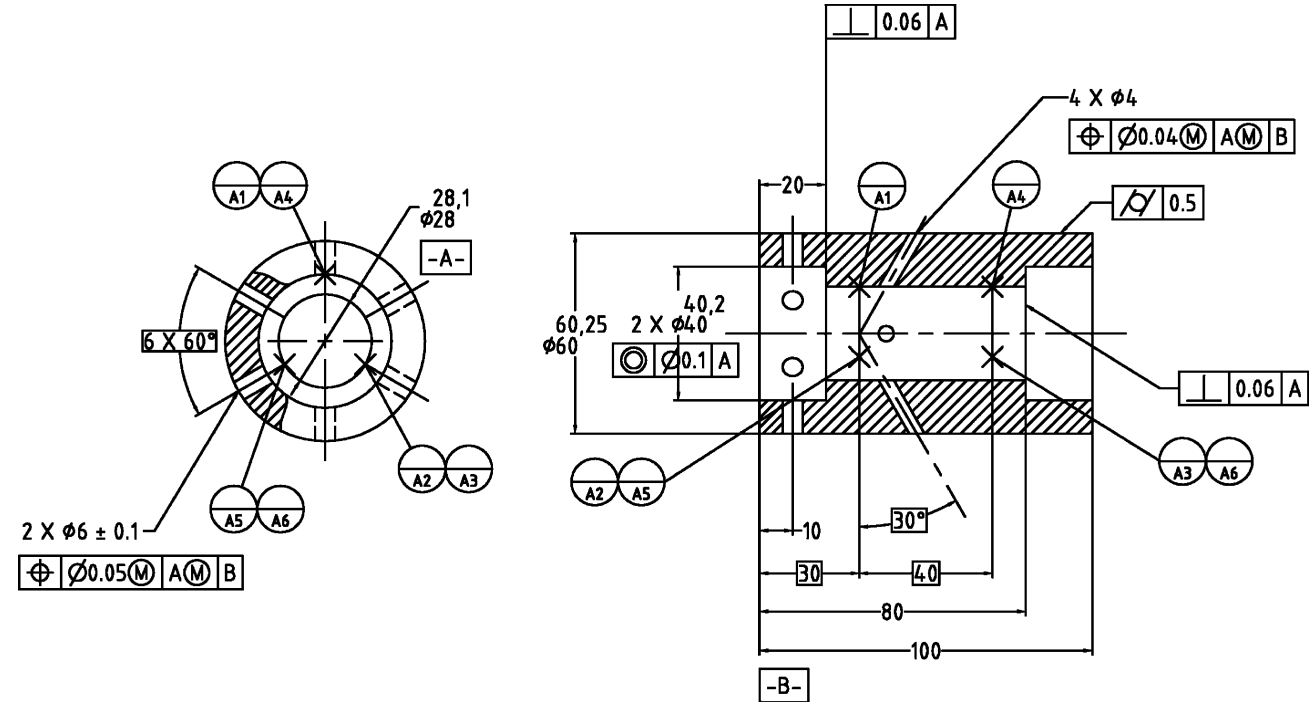

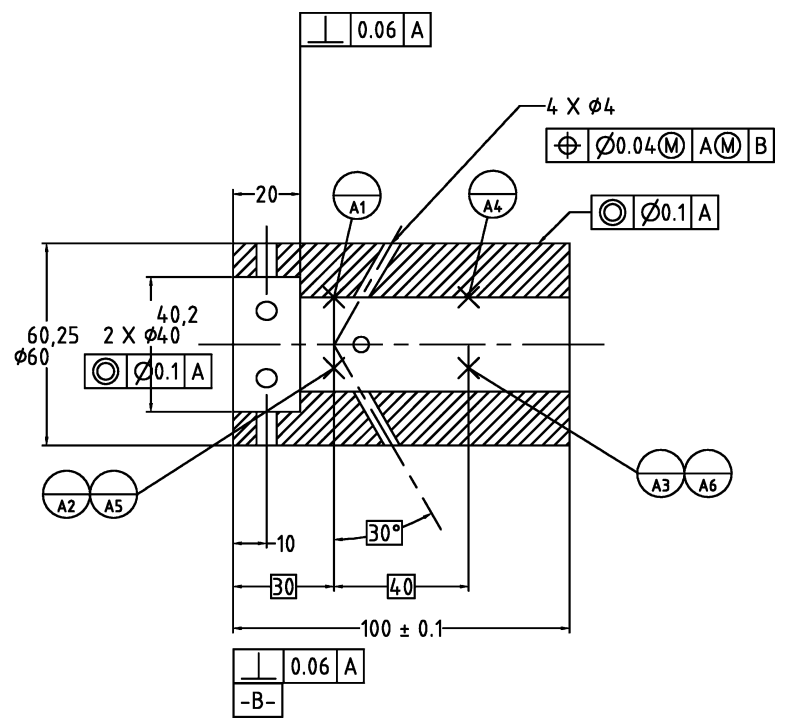

Fig. 16. Original design of product $A$ in example 2
Fig. 17. Original design of product $B$ in example 2

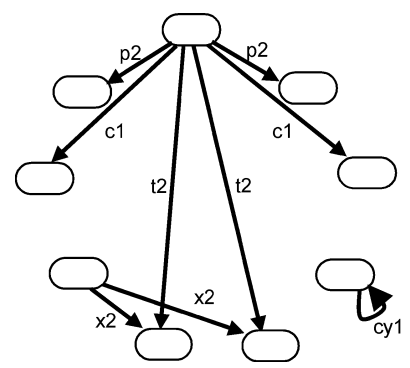

Fig. 18. Constraint networks of products $A$ and $B$ in example 2

datums. The evaluated costs are given in Table 6 and are compared with the result for the original designs with the same production plan variations. The cost of the modified designs shows a $5.9 \%$ cost reduction (see gross cost in Table 6).

Figure 22a-c shows process plans and manufacturing system configurations for three periods and modified designs. As noticed in example 1, example 2 also shows that the higher-volume product dominates the configuration of the manufacturing system, although the processes are not
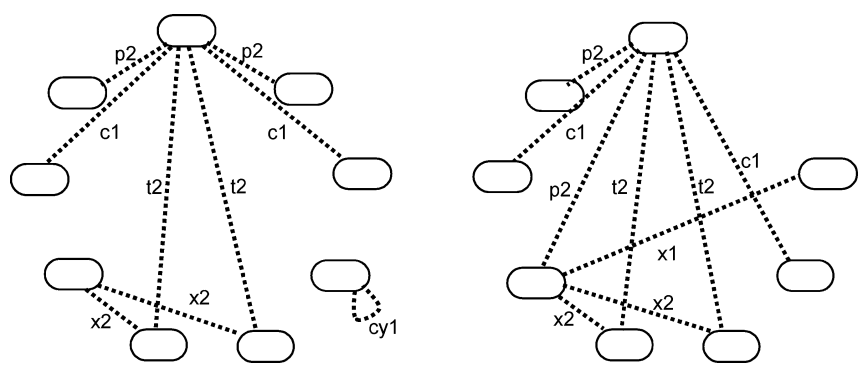

Fig. 19. Representation of functional requirement for example 2

well distributed throughout the manufacturing systems as in example 1.

Figure 25 shows three pairs of products $A$ and $B$ optimized for the production plan of each period. Because the problem is more complex than that in example 1, these $\mathrm{CN}$ do not show as much similarity as those in example 1 . The complexity comes not only from the number of manufacturing features (nodes) but also from the number of constraints (edges). Moreover, the number of datums available for a geometrical tolerance affects the complexity 


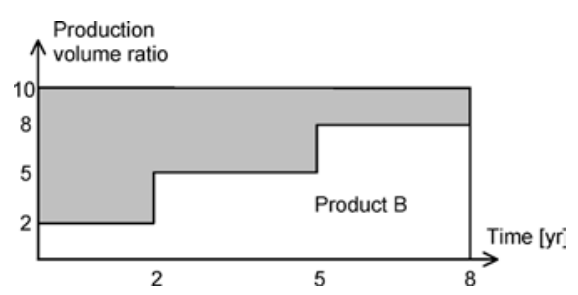

Fig. 20. Production plan variations for example 2

of problem. While only two datums are available for most of the geometrical tolerances in example 1, four or five datums are available in example 2. For example, four features are available (BO1, $\mathrm{BO} 2, \mathrm{BO} 3$ and ST1) for the concentricity (denoted as ' $c 1$ ') in product $A$ of example 2 . In the examples, allowing a specific manufacturing feature to be a datum of a geometrical tolerance was decided based on the shape, size and orientation of the feature. However, experienced engineers could decrease the complexity of the problem by excluding some of the available datums based on their experience. This is very desirable, since GA would find more practical solutions in a shorter time.

\section{6}

\section{Conclusion and discussion}

This paper provides a simple framework to evaluate the manufacturability of a part family and to generate redesign suggestions to reduce the cost for production plan variations. For this purpose, a graphical representation of geometry and tolerance information is devised. Methods to define functional requirements and to generate alternative designs are also presented. An optimization method utilizing GA was developed to find redesign suggestions. Although the results obtained are not guaranteed to be optimum, it shows a certain advantage over original designs that are already fair enough, considering every manufacturing feature and its tolerances are assumed to be part of the functional requirements. However, for more sophisticated products, where more degrees of freedom in defining tolerances and datums exist and the formation of redundant design elements is more probable, the application of this method would provide significant improvement.
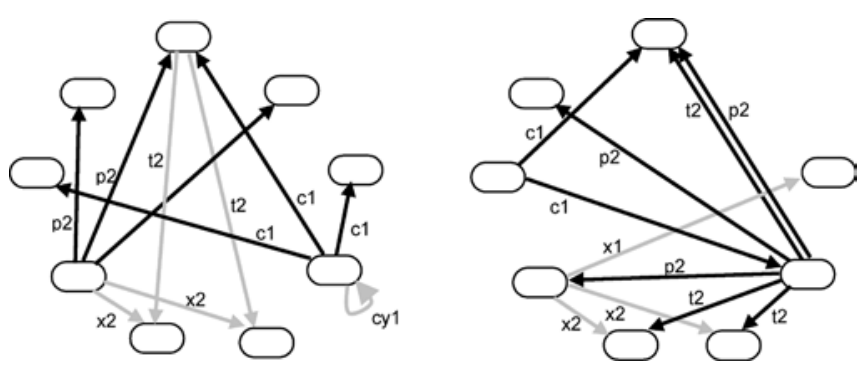

Fig. 21. Modified constraint network of products $A$ and $B$ in example 2

A number of assumptions have been made on the problem formulation. Relaxing these assumptions is the topic of future work, including:

While similar datum definitions may share a fixture, if we set different datum definitions for the same features of different products, a new fixture must be designed and utilized in the manufacturing system, and its cost should be included.

There is also a certain cost for the design changes, although it is not easy to estimate. When a part that has been under production is changed according to a new production plan, changing existing designs will invoke additional overhead cost.

The reconfiguration cost of manufacturing systems between production plans can be included.

For application to more realistic and complex manufacturing systems, the modeling should allow multiple choices of machine for a manufacturing feature to achieve shorter cycle time.

When modifying constraint networks, the effect of datums in terms of tolerance level has been ignored. When a path between a pair of nodes is lengthened, the tolerance level of each edge in the lengthened path should be adjusted higher to meet the tolerance level of the original path because of tolerance accumulation. Then the cost of increasing tolerance level should be estimated and included in overall cost.

Finally, application to assembly design of a product family can be considered in future work, since precedence
Table 4. Material volume to be removed for manufacturing features in example 2

\begin{tabular}{rlllllllll}
\hline $\begin{array}{r}\text { Product A } \\
\text { Feature }\end{array}$ & BO1 & IF1 & BO2 & FA1 & CH1 & TH1 & ST1 & BO3 & IF2 \\
Volume & 30 & 5 & 10 & 8 & 15 & 12 & 60 & 10 & 5 \\
Product B & & & & & & & & & \\
Feature & BO1 & IF1 & BO2 & FA1 & CH1 & TH1 & ST1 & FA2 & - \\
Volume & 30 & 5 & 10 & 8 & 15 & 12 & 60 & 8 & - \\
\hline
\end{tabular}

Table 5. Material removal rate and matching manufacturing features for types of machines in example 2

\begin{tabular}{llll}
\hline Type of machine & Turning (TU) & $\begin{array}{l}\text { Cross-drilling } \\
\text { (CD) }\end{array}$ & $\begin{array}{l}\text { Tapered cross- } \\
\text { drilling (TD) }\end{array}$ \\
\hline MRR & 2 & 4 & 3.5 \\
Manufacturing features & $\begin{array}{l}\text { Straight turning (ST) } \\
\text { Boring (BO) }\end{array}$ & Cross-hole (CH) & Tapered cross-hole (TH) \\
& Facing (FA) & & \\
& Internal facing (IF) & \\
\hline
\end{tabular}




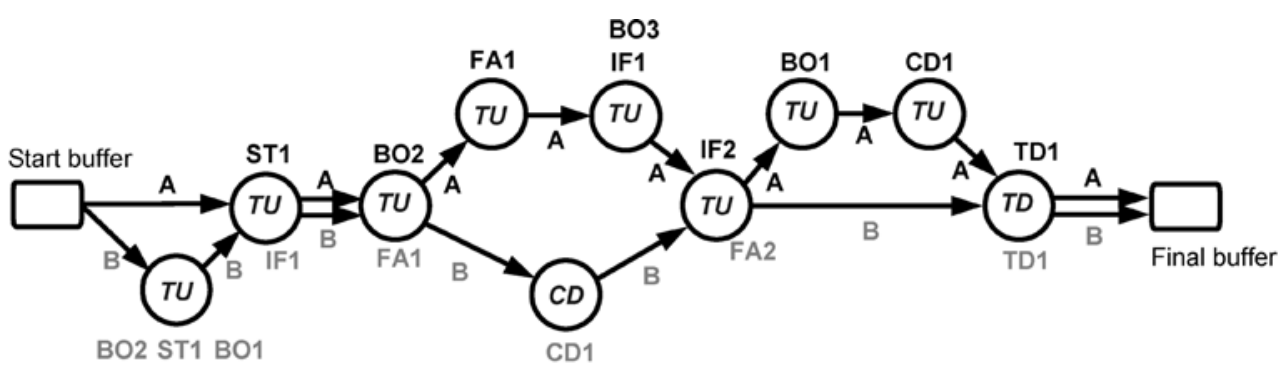

(a) - Config $1, \mathbf{p}_{11}, \mathbf{p}_{21}, \operatorname{Corr}_{11}, \operatorname{Corr}_{21}$ for $\rho\left(\tau_{1}, \mathbf{a}_{1}\right)$

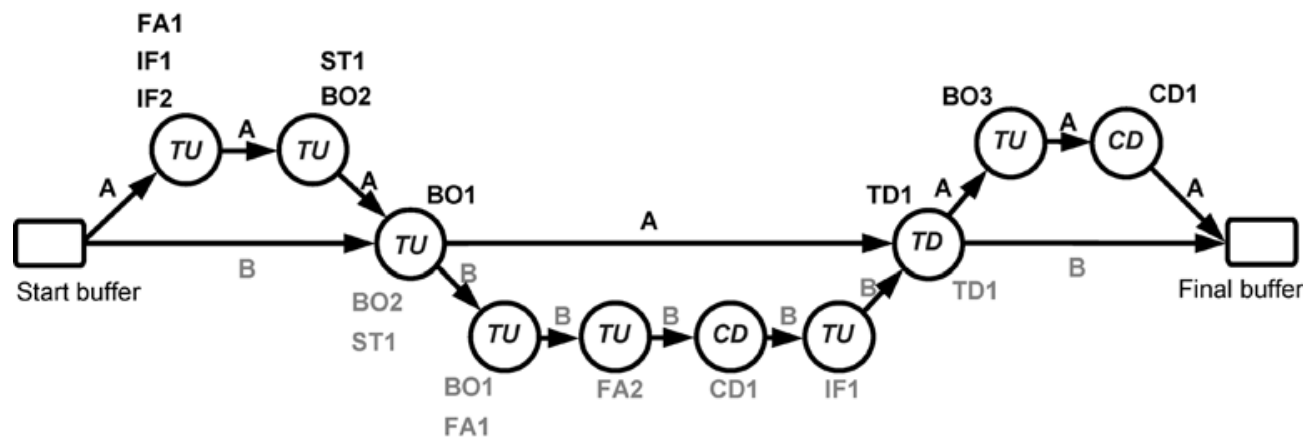

(b) - Config $2, \mathbf{p}_{12}, \mathbf{p}_{22}$, Corr $_{12}$, Corr $_{23}$ for $\rho\left(\tau_{2}, \mathbf{a}_{2}\right)$

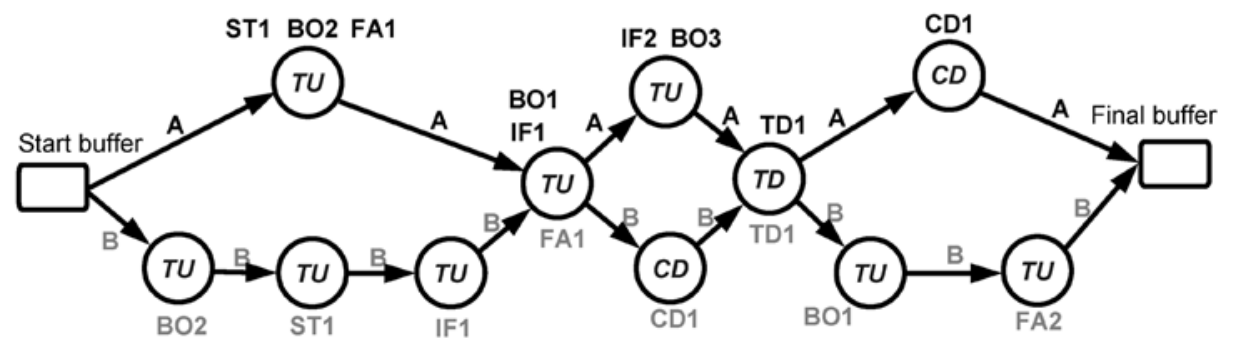

(c) - Config $_{3}, \mathbf{p}_{13}, \mathbf{p}_{23}$, Corr $_{13}$, Corr $_{23}$ for $\rho\left(\tau_{3}, \mathbf{a}_{3}\right)$

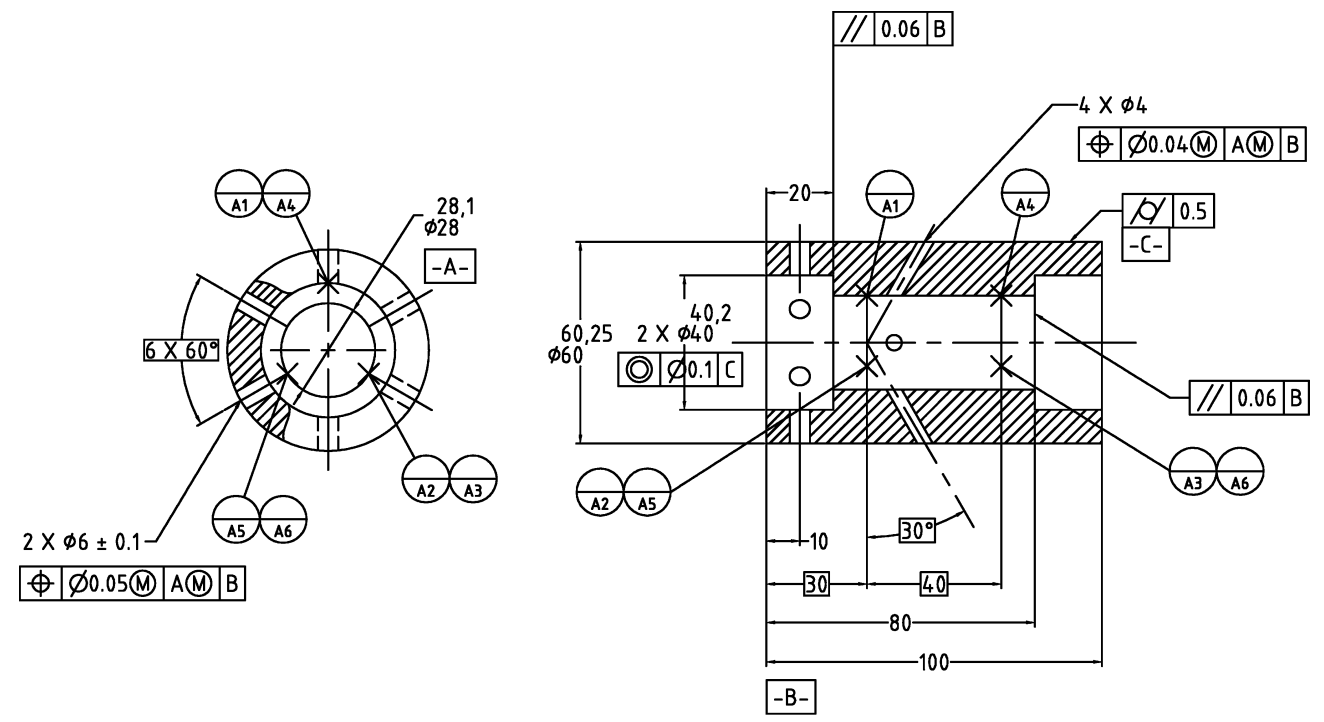

Fig. 22a-c. Process plans and manufacturing system configurations corresponding to three periods of production plans (Fig. 20) and the redesign suggestions (Fig. 21)

Fig. 23. Modified design of product $A$ in example 2 
B. Lee, K. Saitou: Design of part family robust-to-production plan variations based on quantitative manufacturability evaluation

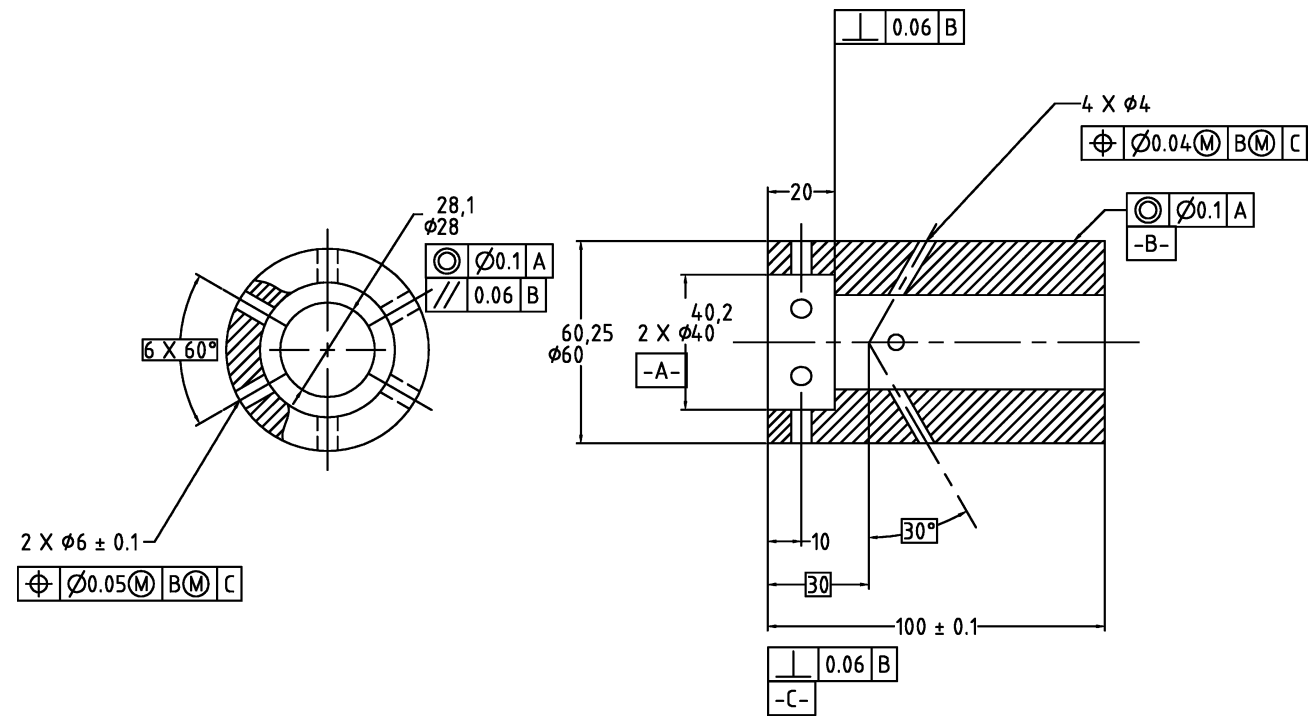

Table 6. Cost comparison between the original and modified designs for example 2

\begin{tabular}{lllll}
\hline & \multicolumn{2}{l}{ Overall cost for period $j$} & & $\begin{array}{l}\text { Gross } \\
\text { cost }\end{array}$ \\
\cline { 2 - 4 } & $j=1$ & $j=2$ & $j=3$ & \\
\hline Original designs & 1122.33 & 1011.22 & 1105 & 8593.32 \\
Modified designs & 1031.56 & 970.667 & 1035.67 & 8082.13 \\
\hline
\end{tabular}
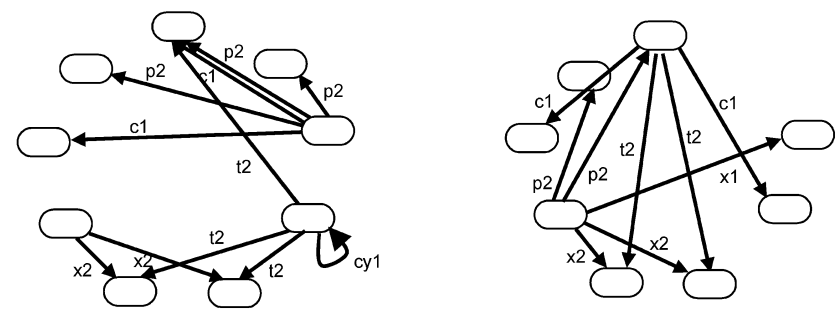

conditions in assembly sequences and core functions must be kept, which is similar to the approach proposed in this research.

\section{References}

Alla H, Ladet P, Martinez J, Silva-Suarez M (1985) Modeling and validation of complex systems by colored Petri nets: application to flexible manufacturing systems. Lecture Notes Comput Sci 188:1-14

(a) - $C N_{1}$ and $C N_{2}$ for period 1
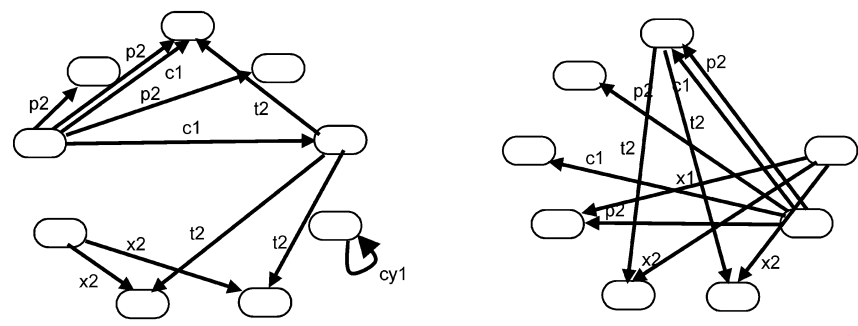

(b) - $C N_{1}$ and $C N_{2}$ for period 2
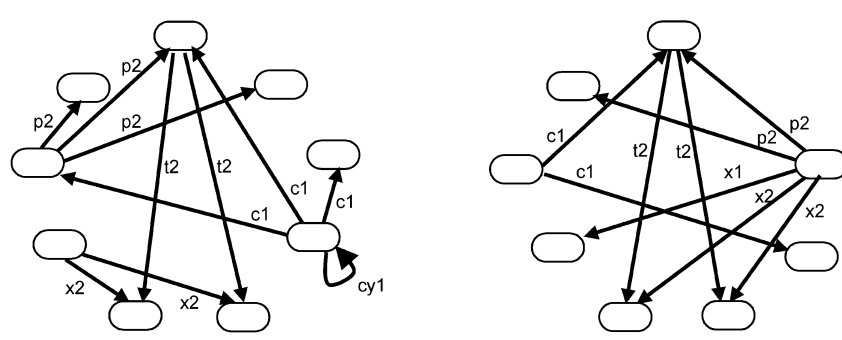

(c) - $C N_{1}$ and $C N_{2}$ for period 3

Fig. 25a-c. Each pair of constraint networks of products $A$ and $B$ is optimized for each volume ratio shown in Fig. 20. For each volume ratio, these $\mathrm{CN}$ give a lower cost than the ones in Fig. 21 optimized throughout the production period

Bralla JG (1999) Design for manufacturability handbook. McGrawHill, New York

Boothroyd G, Dewhurst P, Knight W (1999) Product design for manufacture and assembly. Dekker, New York

Das D, Gupta SK, Nau DS (1994) Reducing setup cost by automated generation of redesign suggestions. In: Proc 1994 ASME International Computers in Engineering Conference and Exhibition, Part 1

Das D, Gupta SK, Nau DS (1996) Generating redesign suggestions to reduce setup cost: a step towards automated redesign. Comput Aided Des 28:763-782

Fu Z, dePennington A (1994) Geometric reasoning based on graph grammar parsing. ASME J Mech Des 116:763-769

Fujita K, Akagi S, Yoneda T, Ishikawa M (1998) Simultaneous optimization of product family sharing system structure and configuration. In: Proc ASME Design Engineering Technical

Conferences, Atlanta, 12-16 September, paper no. DETC98/DFM5722

Goldberg DE (1989) Genetic algorithms in search, optimization and machine learning. Addison-Wesley, Reading, Mass

Gonzalez-Zugasti JP, Otto KN, Baker JD (1998) A method for architecting product platforms with an application to interplanetary mission design. In: Proc ASME Design Engineering Technical Conferences, Atlanta, 12-16 September, paper no. DETC98/DAC5608

Gupta SK, Regli WC, Das D, Nau DS (1997) Automated manufacturability analysis: a survey. Res Eng Des 9:168-190

Hayes CC (1996) Plan-based manufacturability analysis and generation of shape-changing redesign suggestions. J Intell Manuf 7:121132 
Hayes CC, Sun HC (1995) Using a manufacturing constraint network to identify cost-critical areas of designs. Art Intell Eng Des Anal Manuf 9:73-87

Hernandez G, Simpson TW, Allen JK, Bascaran E, Avila LF, Salinas F (1998) Robust design of product families for make-to-order systems. In: Proc ASME Design Engineering Technical Conferences, Atlanta, 12-16 September, paper no. DETC98/DAC-5595

Herrmann JW, Chincholkar MM (2000) Design for production: a tool for reducing manufacturing cycle time. In: Proc ASME Design Engineering Technical Conferences, Las Vegas, 12-15 September, paper no. DETC2000/DFM-14002

Holland JH (1975) Adaptation in natural and artificial systems. Univ of Michigan Press, Ann Arbor

Huang SH, Zhang HC, Oldham WJB (1997) Tolerance analysis for setup planning: a graph theoretical approach. Int J Product Res 35:1107-1124

Ishii K, Juengel C, Eubanks CF (1995) Design for product variety: key to product line structuring. In: Proc ASME Design Engineering Technical Conferences, Boston, 17-20 September, 2:499-506

Joshi S, Chang TC (1988) Graph-based heuristics for recognition of machined features from a 3-D solid model. Comput Aided Des 2:58-66

Kota S, Sethuraman K, Miller R (2000) A metric for evaluating design commonality in product families. J Mech Des 122:403-410

Kusiak A, He DW (1998) Design for agility: a scheduling perspective. Robotics Comput Integrated Manuf 14:415-427

Mehlhorn K, Näher S (1999) The LEDA platform of combinatorial and geometric computing. Cambridge University Press, Cambridge

Madsen DA, Shumaker TM, Turpin JL, Stark C (1991) Engineering drawing and design. Delmar, Albany, New York
Nelson SA II, Parkinson MB, Papalambros PY (1999) Multicriteria optimization in product platform design. In: Proc ASME Design Engineering Technical Conferences, Las Vegas, Paper No. DETC99/DAC-8676

Saitou K, Malpathak S (1999) Robustness optimization of FMS under production plan variations: the case of cyclic production. In: Proc ASME Design Engineering Technical Conferences, Las Vegas, 12-15 September, paper no. DETC99/CIE-9127

Simpson TW, Maier JRA, Mistree F (1999) A product platform concept exploration method for product family design. In: Proc ASME Design Engineering Technical Conferences, Las Vegas, 12-15 September, paper no. DETC99/DTM-8761

Suresh NC, Kay JM (eds) (1998) Group technology and cellular manufacturing: a state-of-the-art synthesis of research and practice. Kluwer, Boston

Tsai J, Cutkosky MR (1997) Representation and reasoning of geometric tolerances in design. Art Intell Eng Des Anal Manuf 11:325-341

Ulrich KT, Eppinger SD (1995) Product design and development. McGraw-Hill, New York

van Vliet JW, van Luttervelt CA, Kals HJJ (1999) State-of-the-art report on design for manufacturing. In: Proc ASME Design Engineering Technical Conferences, Las Vegas, 12-15 September, paper no. DETC99/DFM-8970

Whitney DE (1993) Nippondenso Co Ltd: a case study of strategic product design. Res Eng Des 5:1-20 